\title{
Ectopia renal derecha intratorácica con hernia diafragmática congénita: reporte de un caso y revisión de la bibliografía
}

\section{Intrathoracic right renal ectopia with congenital diaphragmatic hernia: A case report and literature review}

\author{
Axel F Mora-Ramírez, ${ }^{1}$ Rubén Gutiérrez-Rosales, ${ }^{1}$ Marco A Aragón-Castro, ${ }^{1}$ Christian Vázquez-Niño, ${ }^{1}$ Guillermo \\ E Ruvalcaba-Oceguera, ${ }^{1}$ Aldo U Ordoñez-Jurado, ${ }^{1}$ Miguel A Martínez-Arias ${ }^{2}$
}

\begin{abstract}
Resumen
ANTECEDENTES: La ectopia intratorácica denota una protrusión parcial o completa del riñón, a la altura del diafragma, en el mediastino posterior. Es la forma más rara de ectopia renal.

CASO CLíNICO: Paciente femenina de 39 años, que acudió al servicio médico por fiebre de $39^{\circ} \mathrm{C}$, disuria y dolor en la región lumbar derecha tipo cólico, punzante e intermitente, y hemitórax derecho de características similares, náusea y vómito; taquicardia e hipotensión. Entre sus antecedentes personales refirió hipertensión arterial sistémica de 10 años de evolución; hipertensión pulmonar de 13 años y diagnóstico de dextrocardia y situs inversus desde la infancia. En 2005 le diagnosticaron ectopia renal derecha intratorácica, con hernia diafragmática derecha. En relación con su padecimiento actual, la paciente fue ingresada a la unidad de cuidados intensivos por choque séptico de origen urinario. Se realizó nefrectomía derecha con acceso transtorácico y plastia diafragmática. Durante el procedimiento quirúrgico, en la toracotomía derecha, se cuantificaron $500 \mathrm{~mL}$ de líquido purulento en la cavidad torácica, por lo que se realizó lavado con solución fisiológica. También se encontraron múltiples adherencias al riñón ectópico-pleura, se practicó decorticación y se identificó la bolsa hidronefrótica ectópica; se disecó hasta el hilio renal, se ligó y cortó la arteria y vena renal, además del uretero, y se extrajo el riñón ectópico. Posteriormente se llevó a cabo la plastia diafragmática con malla Dualmesh ${ }^{\circledast}$, se colocó un drenaje hacia la cavidad abdominal y una sonda endopleural. La evolución posquirúrgica fue satisfactoria.

CONCLUSIÓN: Los pacientes con ectopia renal pueden o no requerir tratamiento quirúrgico, según su manifestación de síntomas. La bibliografía relacionada con estudios de autopsias o reportes clínicos no señala que los casos diagnosticados con riñón torácico evolucionen a complicaciones urinarias o pulmonares.
\end{abstract}

PALABRAS CLAVE: Hernia diafragmática congénita; ectopia renal; reparación transtorácica.

Abstract

BACKGROUND: Intrathoracic ectopia denotes a partial or complete protrusion of the kidney at the level of the diaphragm in the posterior mediastinum. It is the rarest form of renal ectopia.

CLINICAL CASE: A 39-year-old woman sought medical attention for fever of $39{ }^{\circ} \mathrm{C}$, dysuria, and colicky, stabbing, intermittent pain in the right lumbar region and hemithorax, nausea and vomiting, tachycardia, and hypotension. She had a personal history of high blood pressure of 10-year progression, pulmonary hypertension of 13-year progression, as well as the diagnosis of dextrocardia with situs inversus, since childhood. In 2005, the patient was diagnosed with right intrathoracic renal ectopia with a right diaphragmatic hernia. In relation to her current illness, the patient was admitted to the intensive care unit due to septic shock of urinary origin. Transthoracic right ne-
Departamento de Urología.

2 Departamento de Cirugía de tórax.

Universidad Autónoma del Estado de México, Facultad de Medicina, División de Posgrados, Centro Médico ISSEMyM, Toluca, Estado de México.

Recibido: julio 2018

Aceptado: noviembre 2018

Correspondencia Axel Fabián Mora Ramírez axelm86@hotmail.com

Facebook: Axel F. Mora Ramírez

Este artículo debe citarse como Mora-Ramírez AF, Gutiérrez-Rosales $R$, Aragón-Castro MA, Vázquez-Niño C, Ruvalcaba-Oceguera GE, OrdoñezJurado AU, Martínez-Arias MA. Ectopia renal derecha intratorácica con hernia diafragmática congénita: reporte de un caso y revisión de la bibliografía. Rev Mex Urol. 2018 nov-dic;78(6):458-62. DOI: https://doi.org/10.24245/revmexurol.v78i6.2003 
phrectomy with diaphragmatic repair was performed. During the right thoracotomy, $500 \mathrm{~mL}$ of purulent fluid was observed in the thoracic cavity and so washout with physiologic solution was carried out. Multiple adhesions to the ectopic kidney/pleura were found and decorticated. An ectopic hydronephrotic sac was identified and dissected up to the renal hilum. The renal artery, renal vein, and ureter were ligated and cut and the ectopic kidney was extracted. Diaphragmatic repair was then performed with Dualmesh ${ }^{\circledR}$ and an abdominal cavity drain and intrapleural catheter were placed. The patient's postoperative progression was satisfactory.

CONCLUSIONS: Cases of ectopic kidney may or may not require surgical treatment, depending on their symptomatology. Neither autopsy series nor clinical reports suggest that a thoracic kidney causes serious urinary or pulmonary complications.

KEYWORDS: Congenital diaphragmatic hernia; Renal ectopia; Transthoracic repair.

\section{ANTECEDENTES}

La ectopia intratorácica denota una protrusión parcial o completa del riñón, a la altura del diafragma, en el mediastino posterior. Representa la forma más rara de ectopia renal, se estima que menos de $5 \%$ de los pacientes con ectopia tiene riñón intratorácico, su incidencia varía de 1:13,000 autopsias. ${ }^{1}$

El primer caso de riñón intratorácico fue reportado por Wolfromm en 1940. Pfister-Goedeke y Brunier clasificaron los riñones torácicos en 4 grupos: 1) ectopia renal torácica con diafragma cerrado, 2) eventración del diafragma, 3) hernia diafragmática (defectos congénitos o adquiridos, como la hernia de Bochdalek) y 4) ruptura traumática del diafragma con ectopia renal. ${ }^{2}$

La ectopia renal es un factor predisponente de alteraciones del sistema genitourinario que pueden aparecer por infección de la vía urinaria, sepsis o choque séptico.

\section{CASO CLÍNICO}

Paciente femenina de 39 años, que ingresó al Centro Médico ISSEMyM, Toluca, por cuadro clínico de cinco días de evolución, caracterizado por fiebre de $39^{\circ} \mathrm{C}$, disuria y dolor en la región lumbar derecha tipo cólico, punzante e intermitente, y hemitórax derecho de características similares, náusea y vómito en múltiples ocasiones; taquicardia e hipotensión. El examen general de orina reportó leucocituria y bacteriuria como hallazgos sobresalientes. Entre sus antecedentes personales refirió hipertensión arterial sistémica de 10 años de evolución, en tratamiento (no especificado); hipertensión pulmonar de 13 años de diagnóstico, en tratamiento con bosentán y oxígeno suplementario; diagnóstico de dextrocardia y situs inversus desde la infancia. Como antecedentes quirúrgicos destacó: neumonectomía derecha hace 13 años, secundaria a agenesia de la arteria pulmonar ipsilateral; 1 cesárea hace 12 años por insuficiencia cardiaca materna, histerectomía total abdominal hace 2 años secundaria a miomatosis uterina, y mastoplastia de aumento hace 1 año. En el año 2005 le diagnosticaron ectopia renal derecha intratorácica, con hernia diafragmática derecha, exclusión renal ipsilateral mediante tomografía computada (Figura 1) y gammagrama renal con 99mTc-ácido dietil-triamino-pentacético (DTPA). En relación con su padecimineto actual, la paciente fue ingresada a la unidad de cuidados intensivos por choque séptico de origen urinario, donde permaneció cuatro días, y posteriormente la trasladaron al servicio de Urología y Cirugía de tórax para tratamiento 
quirúrgico. Se realizó nefrectomía derecha con acceso transtorácico (Figura 2) y plastia diafragmática. Durante el procedimiento quirúrgico, en la toracotomía derecha se cuantificaron $500 \mathrm{~mL}$ de líquido purulento en la cavidad torácica, por lo que se realizó lavado con solución fisiológica. También se encontraron múltiples adherencias al

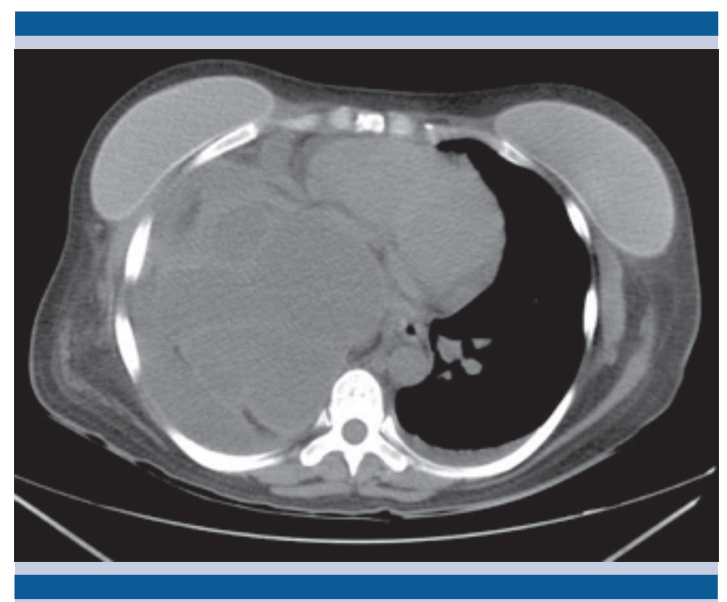

Figura 1. Tomografía simple toracoabdominopélvica en corte axial. En el hemitórax derecho se observa la bolsa hidronefrótica ipsilateral.

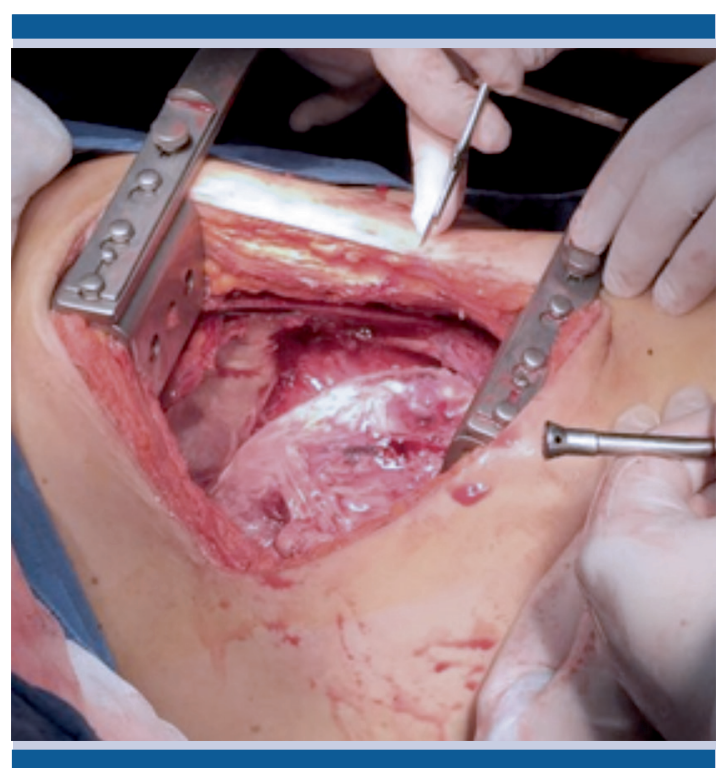

Figura 2. Acceso transtorácico derecho para nefrectomía simple y plastia diafragmática. riñón ectópico-pleura, se practicó decorticación y se identificó la bolsa hidronefrótica ectópica; se disecó hasta el hilio renal, se ligó y cortó la arteria y vena renal, además del uretero, y se extrajo el riñón ectópico. Posteriormente se llevó a cabo la plastia diafragmática con malla Dualmesh ${ }^{\circledR}$, se colocó un drenaje hacia la cavidad abdominal y una sonda endopleural.

La paciente evolucionó satisfactoriamente, sin registro de datos de respuesta inflamatoria sistémica ni dificultad ventilatoria; continuó en vigilancia posquirúrgica hasta el retiro del drenaje y de la sonda endopleural. Desde entonces no ha manifestado signos, síntomas ni complicaciones adicionales.

\section{DISCUSIÓN}

La embriología de las anormalidades congénitas renales implica la participación de importantes componentes: material nefrogénico (pronefros, mesonefros y metanefros) y brotes ureterales (estructuras wolffianas), además de la inducción del tejido metanéfrico (construcción renal) y el ascenso (migración cefálica) y posicionamiento de los riñones (partición y rotación). Los incidentes ocurren durante estas tres fases (primeras 8 semanas de gestación) y pueden afectar la coexistencia, el número y localización de los riñones. $^{3}$

El conducto nefrogénico se divide en tres partes: $\left.{ }^{4} 1\right)$ los segmentos más craneales constituyen el pronefros, 2) los segmentos intermedios el mesonefros y 3) los segmentos más caudales el metanefros o el riñón. ${ }^{5} \mathrm{El}$ pronefros (estado embrionario de 8 somitas) se degenera completamente (día 24-25); sin embargo, su conducto contribuye a la formación del mesonefros. En un embrión con 23 somitas, el mesonefros produce pequeñas cantidades de orina diluida y forma las vesículas mesonéfricas y los túbulos excretores, que también se degeneran. El metanefros se 
desarrolla de la porción nefrogénica caudal del mesonefros. La migración cefálica de los riñones termina en la octava semana de gestación, así como su rotación axial de $90^{\circ}$ medialmente. Durante su ascenso los riñones reciben el aporte sanguíneo de los vasos vecinos. La sangre, inicialmente, llega a través de la arteria sacra media, posteriormente de las arterias mesentéricas común e inferior y finalmente de la aorta.

Las anomalías relacionadas con el ascenso anormal de los riñones se denominan riñones ectópicos e incluyen alteraciones como: riñón pélvico (pélvico o sacro), que representa la localización más común; riñón lumbar o sacro; y riñón torácico, ${ }^{6}$ ésta última es la forma más rara de ectopia renal. Menos de $5 \%$ de los pacientes con ectopia tiene un riñón intratorácico. Su incidencia es de 1:13,000 casos por autopsia. El riñón se sitúa en el mediastino posterior y casi siempre ha completado su proceso normal de rotación. El contorno renal y el sistema colector son normales. Casi siempre, el riñón yace en la cara posterolateral del diafragma, en el foramen de Bochdalek. En este punto el diafragma se adelgaza y forma una membrana delgada que rodea la porción protruyente del riñón. Así pues, el riñón no se encuentra en el espacio pleural. El lóbulo inferior del pulmón adyacente puede estar hipoplásico secundario a una compresión por la masa renal. La vasculatura renal y el uréter entran y salen de la cavidad pleural a través del agujero de Bochdalek. N'Guessan y Stephens determinaron que la glándula suprarrenal ocupa su localización normal y el riñón contralateral no muestra alteraciones. ${ }^{7}$

Las anormalidades asociadas con los riñones ectópicos incluyen alteraciones urinarias contralaterales o genitales como: ausencia de vagina, uréter retrocavo, útero bicorne, riñones supernumerarios y uréter contralateral ectópi$\mathrm{co.}^{2}$ El riñón ectópico puede formar parte de un síndrome más complejo, como el síndrome de
Mayer-Rokitansky-Küster-Hauser, la anemia de Fanconi o gemelos siameses. Los riñones cefalizados también se han reportado en pacientes con onfaloceles. El 56\% de los riñones ectópicos tiene un sistema colector hidronefrótico; la mitad de estos casos resulta de la obstrucción de la unión ureteropiélica o ureterovesical (70 y 30\%, respectivamente), $25 \%$ de reflujo ureterovesical grado III o mayor, y 25\% se debe a mala rotación. El reflujo vesicoureteral se ha encontrado en $30 \%$ de los niños con riñones ectópicos. La incidencia de anormalidades genitales en pacientes con ectopia es de $15 \%$. La mayoría de los pacientes con riñones ectópicos cursan clínicamente asintomáticos, excepto cuando se asocian con uréter ectópico. ${ }^{3}$ El dolor en el flanco fue el síntoma más frecuente en un caso de obstrucción de la unión ureteropiélica y riñón intratorácico. Algunos pacientes pueden padecer hidronefrosis severa, quizá causada por la obstrucción intermitente de las fibras musculares del diafragma. ${ }^{8}$

El diagnóstico de riñón ectópico se establece de forma fortuita y el tratamiento quirúrgico se implementa en función de la manifestación de síntomas, es decir, excreción disminuida o reflujo. Los estudios con isótopos, la ultrasonografía pre y posnatal, la radiografía de tórax, resonancia magnética y urografía intravenosa son efectivos para identificar el parénquima renal ectópico. El diagnóstico puede complementarse con estudios de contraste retrógrados, si se requiere. Incluso, se ha reportado que la tomografía computada puede objetivar la coexistencia de tejido renal ectópico, especialmente en la cavidad torácica. Los casos de riñón ectópico pueden sospecharse prenatalmente cuando la anormalidad se relaciona con algún grado de dilatación. ${ }^{9}$

La bibliografía relacionada con estudios de autopsias o reportes clínicos no señala que los casos diagnosticados con riñón torácico evolucionen a complicaciones urinarias o pulmonares. 


\section{REFERENCIAS}

1. McDougal WS, et al. Campbell-Walsh Urology. $11^{\text {th }}$ ed. Amsterdam: Elsevier, 2016.

2. Pfister-Goedeke L, Brunier E. Intrathoracic kidney in childhood with special reference to secondary renal transport in Bochdalek's hernia. Helv Paediatr Acta 1979;34(4):345-457.

3. Coran A, et al. Pediatric surgery. $7^{\text {th }}$ Ed. London: Elsevier Health Sciences, 2012.

4. Kamble RS, et al. Thoracoscopic repair of renal ectopia associated with congenital diaphragmatic hernia: Report of two cases. African J Urol 2015;21:235-238. DOI: https:// doi.org/10.1016/j.afju.2015.06.002
5. Rouanne M. et al. A rare case of ectopic intrathoracic kidney in a 1-year-old child. Urology 2010;76:57-59. DOI: 10.1016/j.urology.2010.03.009

6. Hirsch MS. Riñón ectópico intratorácico. Caso para diagnóstico. Rev Chil Pediatr 2016;87(3):208-210. DOI: 10.1016/j. rchipe.2015.09.003

7. Subramanian VS, Goldfarb DA. Right-sided intrathoracic renal ectopia with Bochdalek's hernia. Urology 2008;72:1016-1017. DOI: 10.1016/j.urology.2008.05.012

8. Gupta A, et al. Ectopic intrathoracic kidney: A case report and literature review. Hong Kong J Nephrol 2013;15:48e50. DOI: https://doi.org/10.1016/j.hkjn.2013.03.007

9. Murphy JJ, et al. The intrathoracic kidney: should we fix it? J Pediatri Surg 2012;47:970-973. DOI: 10.1016/j.jpedsurg.2012.01.056

\section{AVISO IMPORTANTE}

La Revista Mexicana de Urología se convierte en una publicación solo digital, con todas las ventajas que los medios y dispositivos electrónicos ofrecen. Usted podrá revisar la información mediante el sitio web (www.revistamexicanadeurologia.org.mx) o descargando la app para Android o iPhone.

Para consultar el texto completo de los artículos deberá registrarse por una sola vez con su correo electrónico, crear una contraseña, indicar su nombre, apellidos y especialidad.

Esta información es indispensable para saber qué consulta y cuáles son sus intereses, y poder en el futuro inmediato satisfacer sus necesidades de información. 